\title{
In vitro genetics of barley (Hordeum vulgare L.): Response of immature embryos to 2,4-dichlorophenoxyacetic acid
}

\author{
W. Powell* and \\ J. M. Dunwell†
}

\author{
* Scottish Crop Research Institute, Pentlandfield, \\ Roslin, Midlothian, EH25 9RF, U.K. \\ † John Innes Institute, Colney Lane, \\ Norwich, NR4 7UH, U.K.
}

This study involved the response of immature embryos of nine cultivars of spring barley to culture on media containing 2,4-dichlorophenoxyacetic acid (2,4-D). Each embryo, with any attached callus, was harvested after 28 days culture and its shoot length, root length, fresh weight, dry weight and water content was recorded. For each character, there were significant differences between genotypes, between 2,4-D concentrations and also significant genotype $x$ medium interactions. These interactions were successfully analysed using either a joint regression analysis, or a genotype's phenotypic variance over the range of 2,4-D concentrations. This two dimensional assessment of the response of a genotype to culture environment may be useful in defining more critically the parameters involved in the control of barley cell proliferation in vitro.

\section{INTRODUCTION}

The genetic manipulation of crop plants in vitro depends upon the development of appropriate cell and tissue culture systems. Many recent studies on cereals have involved the use of either immature or mature embryos as a source of such cultures and regeneration of entire plants from these tissues has been achieved in a number of species including Zea mays (Green and Phillips, 1975), Sorghum bicolor (Dunstan et al., 1978), Triticum aestivum (Lazar et al., 1983) and Hordeum vulgare (Hanzel et al., 1985; Thomas and Scott, 1985). Unfortunately, much of the work has described the kind and extent of callus growth and proliferation in a qualitative rather than a quantitative manner and hence it is difficult to make detailed comparisons between different investigations.

Bayliss and Dunn (1979) examined the ability of 45 barley cultivars to form callus in response to the synthetic auxin 2,4-dichlorophenoxyacetic acid (2,4-D), and scored the growth of callus, roots and shoots on an arbitrary $0-4$ scale. An alternative method of estimation was adopted by Dale and Deambrogio (1979), who used callus area as an estimate of callus growth. These authors found differences in the amount of callus formation between the two cultivars Akka and Himalaya. In the most recent study (Goldstein and Kronstad,
1986), which involved 20 spring barley genotypes, callus fresh weight was used as an estimate of response in vitro.

The interaction of genotype with 2,4-D or other components of the media has rarely been considered in barley tissue culture experiments (Dunwell, 1981 $a, b, 1983$; Hanzel, 1985). Dunwell (1981 b) found a distinct difference in the responses of mature embryos of two genotypes of barley to concentrations of 2,4-D and sucrose, and to incubation temperature. The present study examined the response of immature embryos of these two genotypes as well as a number of other spring barley cultivars to 2,4-D concentration.

\section{MATERIALS AND METHODS}

\section{Experimental material}

The following spring barley cultivars were used in this study: Apex, Dissa, Doublet, Glacier, Golden Promise, Heriot, Nairn, R23 and 4082. The first seven genotypes are commercial cultivars obtained from the SCRI cereal museum. R23 and 4082 are multiple marker genetic stocks produced by Dr: R. I. Wolfe, Agriculture Canada Research Station, Box 610, Brandon, Manitoba R7A 527, Canada but obtained from the Jonn Innes Institute, Norwich, England. The plants were grown in a glass- 
house with a minimum $16 \mathrm{~h}$ photoperiod at a temperature of $20-30^{\circ} \mathrm{C}$.

\section{Culture of immature zygotic embryos}

One plant was used from each genotype and a number of spikes harvested. Caryopses containing embryos 1-2 $\mathrm{mm}$ in length were removed from the spike and randomised, in order to minimise any effect of developmental stage along the spike. For the six row genotypes (Dissa, Glacier and 4082) only embryos from the primary florets were excised and cultured. Caryopses were sterilised for $15 \mathrm{mins}$ in $2 \%$ sodium hypochlorite with Tween 20 to act as a wetting agent and were then washed in sterile distilled water. Embryos were excised and placed with the scutellum uppermost on $20 \mathrm{ml}$ of solidified medium (agar 0.7 per cent) at a frequency of five per petri dish $(90 \mathrm{~mm} \times 15 \mathrm{~mm})$. The medium employed was based on that of Murashige and Skoog (1962) with the major salts at half the recommended concentration, 3 per cent sucrose, and 2,4-D at 1, 2, 4 or $8 \mathrm{mg} \mathrm{l}^{-1}$. Dishes were sealed with parafilm and incubated at $20^{\circ} \mathrm{C} \pm 2^{\circ} \mathrm{C}$ with a $16 \mathrm{~h}$ plotoperiod provided by white fluorescent tubes $\left(30 \mu \mathrm{E} \mathrm{m}^{-2} \mathrm{~s}^{-1}\right)$.

\section{Experimental design and statistical analyses}

After 28 days in culture, each embryo and attached callus was harvested and its shoot and root length recorded. After blotting with paper tissues the fresh weight was then recorded. After drying overnight at $35^{\circ} \mathrm{C}$, the dry weight was recorded and subsequently the water content calculated.

The experiment was a randomised complete block design with two replicates. Each genotype was represented by 10 embryos within each environment (concentration of 2,4-D) and genotype was considered a fixed effect. Environment was also regarded as a fixed effect and the following fixed effects model (Model 1) was used for the analysis of variance.

$$
Y_{i j k}=\mu+G_{i}+E_{j}+G E_{i j}+B_{j(k)}+e_{i j(k)}
$$

where: $Y_{i j k}$ is the response of the $i$ th genotype in the $k$ th block within the $j$ th environment, $\mu=$ mean response, $G_{i}=$ effect due to the $i$ th genotype, $E_{j}=$ effect due to the $j$ th environment (or concentration of 2,4-D), $G E_{i j}=$ effect due to the interaction of the $i$ th genotype with the $j$ th environment (genotype $\times$ medium interaction). $B_{j(k)}=$ effect due to the $k$ th block in the $j$ th environment, $e_{i j(k)}=$ random sampling component.
The components of variance $\sigma_{G}^{2}, \sigma_{E}^{2}, \sigma_{G E}^{2}, \sigma_{B}^{2}$ and $\sigma_{e}^{2}$ were calculated from the expected mean squares appropriate to the model. Where the genotype $\times$ medium interactions were significant, a joint regression analysis was carried out (Yates and Cochran, 1938) and the interactions partitioned into a heterogeneity of regression item and a deviation from regression item. The regression item was tested for significance against the deviation from regression item when the latter was significantly larger than the error item. For each genotype the phenotypic variance to increasing concentrations of 2,4-D was also used to assess its in vitro sensitivity (Jinks and Pooni, 1980).

\section{RESULTS}

The mean values recorded for each character are given in table 1 . The degree of germination of the embryos is estimated by the two characters, shoot length and root length, both of which tend to show a progressive reduction with increasing 2,4-D concentration. With one exception (water content for 4082) all genotypes show the highest values for the other three characters on media with 2 or $4 \mathrm{mg} \mathrm{l}^{-1} 2,4-\mathrm{D}$. Analysis of variance (table 2 ) shows that the effect of genotype, 2,4-D concentration and the interaction of genotype with $2,4-\mathrm{D}$ concentration were significant $(P<0.05)$ for all the characters. Estimates of the components of variance calculated from the expected mean squares are shown in table 3 and provide estimates of the contributions made by different sources to the variance of the measurements made. These components have been scaled to 100 in order to facilitate comparisons between characters. The table shows that for the three each characters, fresh weight, dry weight and water content, the $\sigma_{G E}^{2}$ component is much greater than the $\sigma_{E}^{2}$ component (i.e., interaction of genotype with 2,4-D is of greater importance than the concentration of $2,4-\mathrm{D}$ ). Indeed, in the case of dry weight the $\sigma_{G E}^{2}$ component accounts for most $(54.7$ per cent) of the variation in the experiment. For the two characters, fresh weight and water content, $\sigma_{G}^{2}$ (i.e., difference between genotypes) accounts for the largest part of the variation, and for shoot length and root length, the experimental error item is the greatest source of variation.

Neither a logarithmic nor a square root transformation of the data was successful in removing the interaction item, suggesting that it is not a scalar effect. This item was therefore analysed further by joint regression analysis (table 4). This 
Table 1 Mean values for the characters: shoot length, root length, fresh weight, dry weight and water content

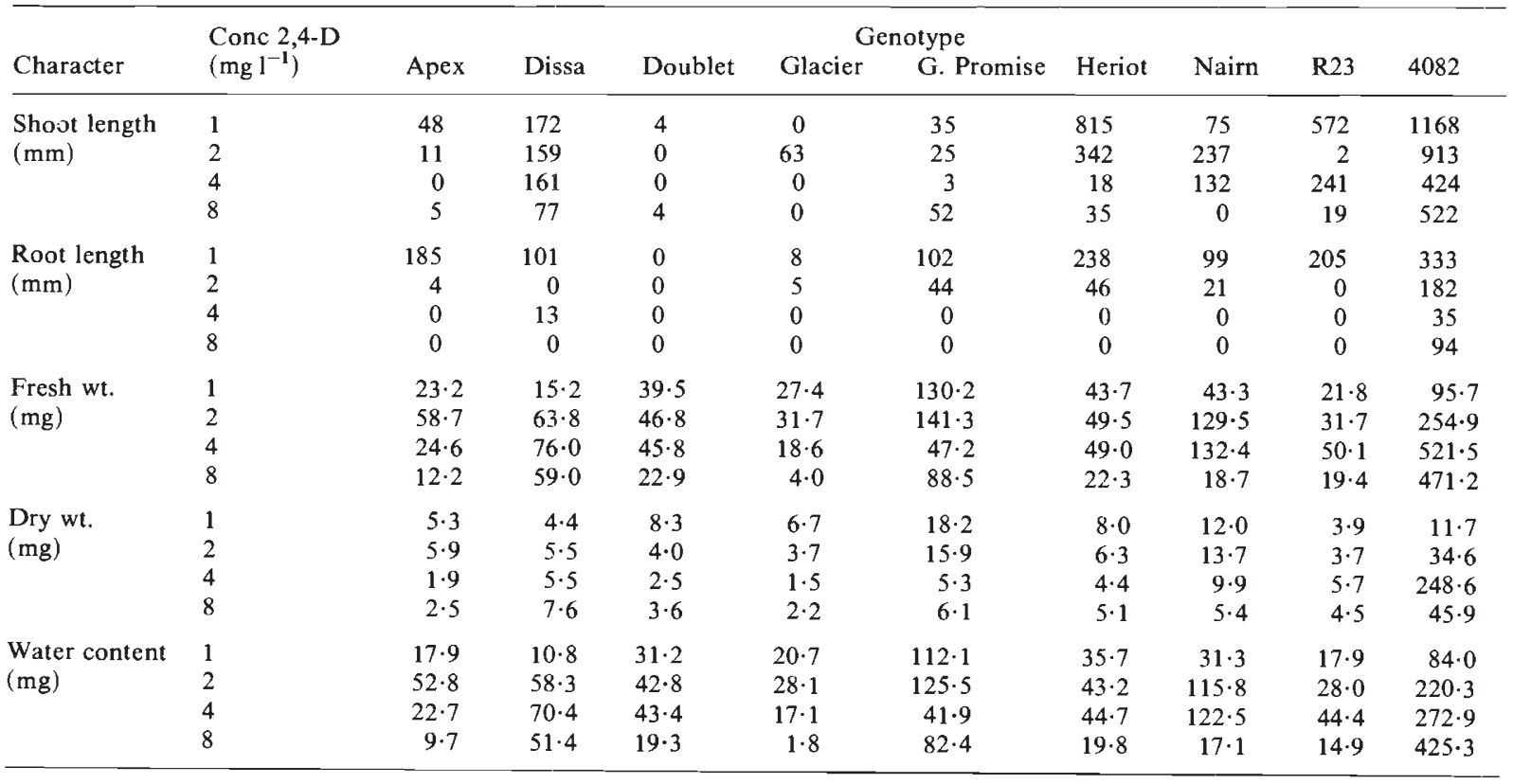

Table 2 Analyses of variance for the characters: shoot length, root length, fresh weight, dry weight and water content

\begin{tabular}{|c|c|c|c|c|c|c|}
\hline Item & df & $\begin{array}{l}\text { Shoot length } \\
\text { MS }\left(10^{-4}\right)\end{array}$ & $\begin{array}{l}\text { Root length } \\
\text { MS }\left(10^{-2}\right)\end{array}$ & $\begin{array}{l}\text { Character } \\
\text { Fresh wt. } \\
\text { MS }\left(10^{-3}\right)\end{array}$ & $\begin{array}{l}\text { Dry wt. } \\
\operatorname{MS}\left(10^{-3}\right)\end{array}$ & $\begin{array}{l}\text { Water cont. } \\
\text { MS }\left(10^{-3}\right)\end{array}$ \\
\hline 2. Blocks within conc & 4 & $7 \cdot 32$ & $28 \cdot 53$ & $7 \cdot 53$ & 0.06 & $7 \cdot 57$ \\
\hline 3. Genotype & 8 & $205 \cdot 90^{* * *}$ & $922 \cdot 49^{* * *}$ & $329 \cdot 49 * * *$ & $28 \cdot 02^{* *}$ & $212 \cdot 89^{* * *}$ \\
\hline 4. Genotype $\times$ Medium & 24 & $28 \cdot 90^{* * *}$ & $239 \cdot 48^{* * *}$ & $50 \cdot 97^{* * *}$ & $13 \cdot 78^{* * *}$ & $28 \cdot 76^{* * *}$ \\
\hline
\end{tabular}

$\dagger$ Indicates number of missing values.

$* P<0.05, * * P<0 \cdot 01, * * * P<0.001$.

technique, which was developed and advanced by Finlay and Wilkinson (1963) and Perkins and Jinks $(1968 a, b)$, involves quantifying each environment (i.e., concentration of $2,4-\mathrm{D}$ ) by the means of all the genotypes in the experiment. For each genotype the linear regression of individual values on the

Table 3 Components of variance scaled to sum to 100

\begin{tabular}{lrlrrr}
\hline & & \multicolumn{4}{c}{ Character } \\
Item & $\begin{array}{c}\text { Shoot } \\
\text { length }\end{array}$ & $\begin{array}{l}\text { Root } \\
\text { length }\end{array}$ & \multicolumn{1}{l}{$\begin{array}{l}\text { Fresh } \\
\text { wt. }\end{array}$} & Dry wt. & $\begin{array}{l}\text { Water } \\
\text { content }\end{array}$ \\
\hline$\sigma_{G}^{2}$ & 33.38 & 16.19 & 44.62 & 27.94 & 43.43 \\
$\sigma_{E}^{2}$ & 14.06 & 29.72 & 2.90 & 4.86 & 2.33 \\
$\sigma_{G E}^{2}$ & 15.59 & 13.85 & 25.33 & 54.66 & 20.63 \\
$\sigma_{B}^{2}$ & 0.30 & 0.00 & 0.33 & 0.00 & 0.70 \\
$\sigma_{e}^{2}$ & 36.71 & 40.24 & 26.81 & 12.55 & 32.85 \\
\hline
\end{tabular}

four media means is calculated. The salient feature of this analysis is that the media are measured in biological terms. The sums of squares measuring the interaction of genotype with medium may be partitioned into an heterogeneity term which measures the differences between the slopes of the regressions and a deviation term which measures the scatter of points about the regression lines.

Considering the character dry weight, the heterogeneity of regression was highly significant when tested against the deviations from regression and therefore accounted for much of the interaction. Thus the linear model will have considerable predictive value in determining the response of a genotype to increasing concentrations of 2,4-D. In other words, a major part of the genotype $x$ medium interaction variance is explained by 
Table 4 Joint regression analyses

\begin{tabular}{|c|c|c|c|c|}
\hline Character & $\begin{array}{l}\text { Genotype } \\
\times \text { medium }\end{array}$ & $\begin{array}{l}\text { an squares and } \\
\text { Heterogeneity } \\
\text { of } \\
\text { regression } \dagger\end{array}$ & $\begin{array}{l}\text { ficance level } \\
\text { Deviations } \\
\text { from } \\
\text { regression }\end{array}$ & Error \\
\hline Shoot length & $28 \cdot 90^{\text {***** }}$ & $62 \cdot 56^{* *}$ & $12 \cdot 06^{* *}$ & $5 \cdot 51$ \\
\hline Root length & $239 \cdot 48^{* * * *}$ & $625 \cdot 57^{* * *}$ & $46 \cdot 43$ & $53 \cdot 93$ \\
\hline Fresh weight & $50 \cdot 97^{* * *}$ & $82 \cdot 18$ & $35 \cdot 37 * * *$ & $4 \cdot 88$ \\
\hline Dry weight & $13 \cdot 78^{* * *}$ & $40 \cdot 55^{* * *}$ & $0 \cdot 40$ & 0.31 \\
\hline Water content & $28 \cdot 76^{* * *}$ & $28 \cdot 23$ & $29 \cdot 03^{* * *}$ & $4 \cdot 00$ \\
\hline
\end{tabular}

† Tested against deviations from regression item.

** $P<0.01$.

$* * * P<0.001$.

differences between the slopes of the linear regressions for the character dry weight. The actual regression lines with their coefficients and standard errors for the multiple marker stocks 4082 and R23 are given in fig. 1 which demonstrates that 4082 embryos produce more callus in response to increasing concentrations of 2,4-D. In contrast, R23 embryos do not show such sensitivity to 2,4-D.

With respect to fresh weight and water content, the deviations mean square is significantly greater than the error mean square. In these cases the deviation from linearity is not due to experimental error and thus the linear regression approach will have little predictive value. An alternative measure of the sensitivity of a genotype to increasing 2,4-D concentration is therefore required. The square root of the variance $(\sigma)$ component over con-

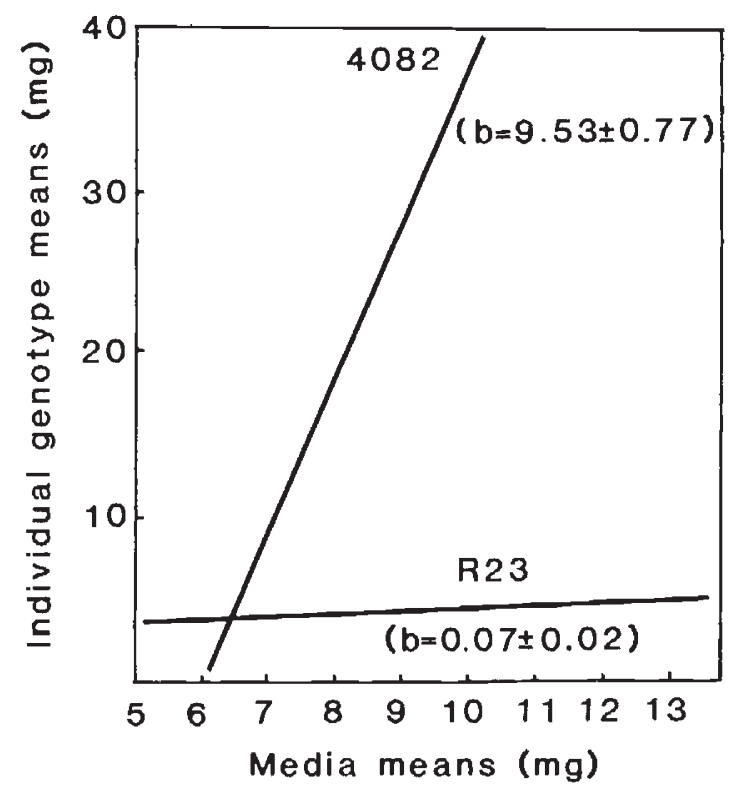

Figure 1 Regression of genotype performance (R23 and 4082) on media means for the character dry weight. centrations of 2,4-D may be used and the analyses of variance based on $\sigma$ for three of the characters scored are given in table 5 . For each character the

Table 5 Analyses of variance for the sensitivity $(\sigma)$ of genotypes to $2,4-\mathrm{D}$

\begin{tabular}{|c|c|c|c|c|}
\hline Item & $\mathrm{dt}$ & $\begin{array}{l}\text { Fresh wt. } \\
\text { MS }\end{array}$ & $\begin{array}{l}\text { Character } \\
\text { Dry wt. } \\
\text { MS }\end{array}$ & $\begin{array}{l}\text { Water } \\
\text { content } \\
\text { MS }\end{array}$ \\
\hline $\begin{array}{l}\text { 1. Between } \\
\text { reps }\end{array}$ & 1 & 290.48 & 0.003 & $541 \cdot 75$ \\
\hline $\begin{array}{l}\text { 2. Between } \\
\text { genotypes }\end{array}$ & 8 & $5327 \cdot 40^{* * * *}$ & $2545 \cdot 30^{* * *}$ & $3515 \cdot 39^{* * *}$ \\
\hline $\begin{array}{l}\text { 3. Reps } x \\
\text { genotypes }\end{array}$ & 8 & $219 \cdot 1$ & $2 \cdot 12$ & $243 \cdot 19$ \\
\hline
\end{tabular}

between genotypes item is highly significant $(P<$ $0 \cdot 001)$ and thus indicates that the genotypes differ in their response to increasing concentrations of 2,4-D. Furthermore, these differences would appear to be under genetic control. There are therefore two genetical aspects of a genotype's phenotypic expression on different culture media. These two statistics, first, mean performance averaged over all concentrations of 2,4-D, and secondly, variation in performance over these environments, are given in table 6. This two dimensional assessment of performance provides a convenient way of ranking a genotype's response to culture and may also be used for further biometrical analyses.

\section{DISCUSSION}

The advantage of the quantitative approach adopted in the present study is that it enabled, for the 
Table 6 Mean $(\bar{x})$ and square root of the variance $(\sigma)$ component over four concentrations of 2,4-D

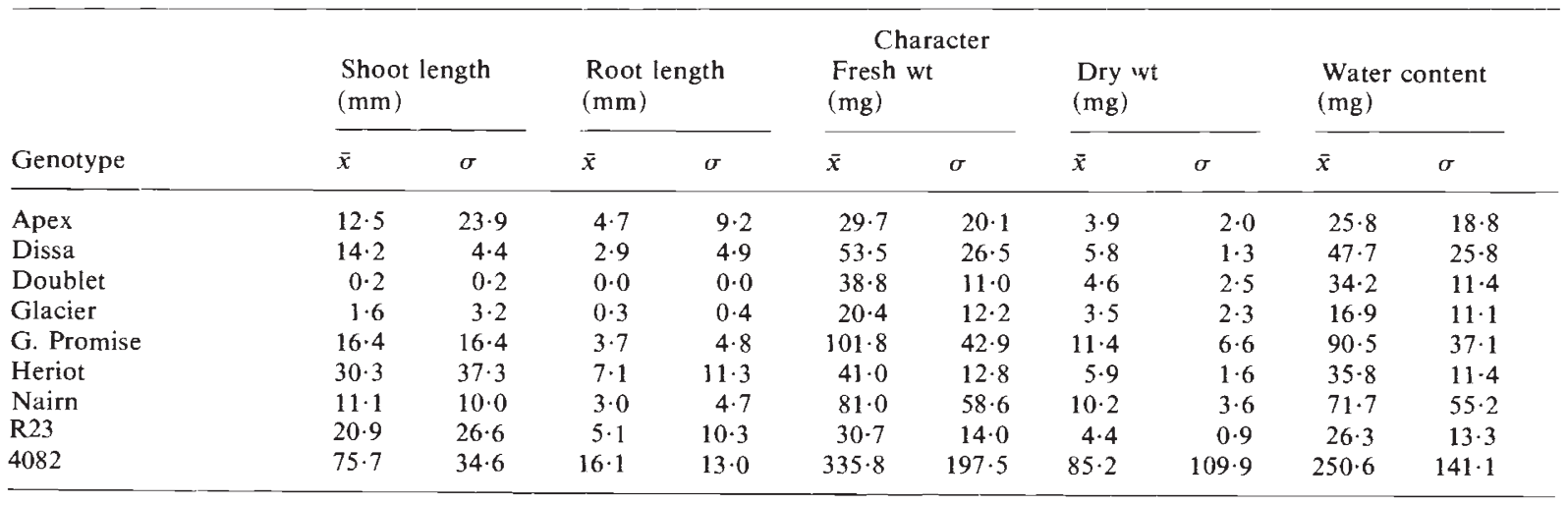

first time, the relative importance of $2,4-\mathrm{D}$, genotype and the interaction between them to be accurately assessed. For example, in the case of dry weight, 55 per cent of the variation is accounted for by the interaction of genotype with 2,4-D (table 3 ). Hence any study of in vitro response in barley, and probably other species, must consider both the mean performance of each genotype and also its response, or sensitivity, to media constituents such as 2,4-D. Analysis of this in vitro response using the joint regression approach and the square root of the variance component taken over a range of 2,4-D concentration has not been reported previously. Furthermore, the analysis described may be extended to include more than one factor. For example, carbohydrate source, incubation temperatures and illumination could be varied and incorporated into factorially designed experiments. This would allow the relative importance of each factor to be quantified and the joint regression analysis would allow genotype by media interaction to be interpreted. In the absence of a significant linear relationship, a genotype's phenotypic variance over culture treatments may be used to measure sensitivity. Such an approach would have the added advantage that the genetics of sensitivity to response in vitro could be investigated.

The first study to compare the growth of embryos from a number of barley genotypes in a range of in vitro environments was that of Dunwell $(1981 b)$ who used the two multiply marked genotypes 4082 and R23. Mature embryos were cultured, scutellum upwards, on media containing a series of combinations of 2,4-D and sucrose and incubated at a range of temperatures. This study showed that at $20^{\circ} \mathrm{C}$ (the temperature used in the present study) 2,4-D (2, 4 or $\left.8 \mathrm{mg} \mathrm{l}^{-1}\right)$ greatly inhibited shoot length although it had little effect: on either dry weight or water content of embryos of either genotype. The significant effects of 2,4-D found in the present study for the two latter charac. ters are presumably due to the greater sensitivity of immature embryos. The previous study (Dunwell, 1981b) did, however, show significant genotype $\times$ environment interactions when the effects of sucrose content and incubation temperature were considered.

The study by Hanzel et al. (1985) was the first to describe a genotype $\times$ medium interaction for the response of immature barley embryos in vitro. There was evidence of an interaction between genotype and basic salt components as well as between genotype and 2,4-D concentration over the range from 0.5 to $2 \mathrm{mg} \mathrm{l}^{-1}$ for the number of embryos with callus. In particular, the cultivar Amsel showed a threefold increase in the frequency of responsive embryos over this range, while the genotype X2387-3 showed a four fold reduction over the same range. It must be noted, however, that this study differed from the present one in that the embryos were placed with the scutellum in contact with the medium. Embryo orientation has been reported to have a significant effect on the frequency and amount of callus produced from immature embryos (Dale and Deambrogio, 1979; Powell, Hayter and Wood, 1983), although this was not found to be so in the study of Goldstein and Kronstad (1986).

Other aspects of the results from the present study can also be compared with those from similar previous investigations. In the study of Hanzel et al. (1985), 30 genotypes were examined and amongst the conclusions reached was that two rowed cultivars showed higher numbers of immature embryos with callus than did six rowed ones. The authors suggested that two rowed types might therefore be more amenable to tissue culture. 
In the present study, however, there is no evidence of such a distinction. Indeed, two of the genotypes show the opposite response in that the six rowed type (4082) produced many times more callus than the two rowed type (R23) and was in fact the most responsive of all the genotypes tested.

These two particular stocks will be used in future genetical studies to assess the effects of major genes on in vitro development in barley (Jacobsen, 1976). Furthermore, the relationship between callus initiation, callus proliferation and shoot differentiation will be investigated (Goldstein and Kronstad, 1986). This aspect of in vitro development is important since it will determine whether or not these responses are under separate genetic control and hence amenable to independent adjustment by suitable selection procedures.

Acknowledgements We acknowledge the technical assistance of Mrs V. Goodall.

\section{REFERENCES}

BAYLISS, M. W. AND DUNN, S. D. M. 1979. Factors affecting callus formation from embryos of barley (Hordeum vulgare). Pl. Sci. Lett., 14, 311-316.

DALE, P. J. AND DEAMBROGIO, E. 1979. A comparison of callus induction and plant regeneration from different explants of Hordeum vulgare. Z. Pflanzenphys, 94, 65-77.

DUNSTAN, D. I., SHORT, K. L. AND THOMAS, E. 1978. The anatomy of secondary morphogenesis in cultured scutellum tissues of Sorghum bicolor. Protoplasma, 92, 251-260.

DUNWELL, J. M. 1981a. Dormancy and germination in embryos of Hordeum vulgare L. Effect of dissection, incubation temperature and hormone application. Ann. Bot., 48, 203213.

DUNWELL, J. M. 1981 b. Influence of genotype and environment on growth of barley embryos in vitro. Ann. Bot., 48, 535-542.
DUNWELL, J. M. 1983. Development of zygotic barley embryos in vitro. In Sen, S. K. and Giles, K. L. (eds.) Plant Cell Culture in Crop Improvement. Plenum, New York, pp. 89 103.

FINLAY, K. W. AND WILKINSON, G. N. 1963. The analysis of adaptation in a plant breeding programme. Aust. J. Agric. Res., 14, 742-754.

GOLDSTEIN, C. S. AND KRONSTAD, W. E. 1986. Tissue culture and plant regeneration from immature embryo explants of barley, Hordeum vulgare. Theor. Appl. Genet., 81, 631-636.

GREEN, C. E. AND PHILI.IPS, R. L. 1975. Plant regeneration from tissue cultures of maize. Crop Sci., 15, 417-421.

HANZEL, J. J., MILLER, J. P., BRINKMAN, M. A. AND FENDOS, E. 1985. Genotype and media effects on callus formation and regeneration in barley. Crop Sci., 25, 27-31.

JACOBSEN, H. J. 1976. Genotypic and environmental influences on germination and callus induction in Hordeum distichum. Bioch. Physiol. Pflanzen, 169, 453-460.

JINKS, J. L. AND POONI. H. S. 1980. Comparing predictions of mean performance and environmental sensitivity of recombinant inbred lines based upon $F_{3}$ and triple test cross families. Heredity, 45, 305-312.

LAZAR, M. D., COLlinS, G. B. AND VIAN, W. E. 1983. Genetic and environmental effects on the growth and differentiation of wheat somatic cell cultures. $J$. Hered, 74, 353-357.

MURASHIGE, T. AND SKOOG, F. 1962. A revised medium for rapid growth and bioassays with tobacco tissue cultures. Physiol. Planta., 15, 473-497.

PERKINS, J. M. AND JINKS, J. L. $1968 a$. Environmental and genotype-environmental components of variability. III. Multiple lines and crosses. Heredity, 23, 339-356.

PERKINS, J. M. AND JINKS, J. L. $1968 b$. Environmental and genotype environmental components of variability. IV. Non-linear interactions of multiple inbred lines. Heredity, 23, 525-535.

POWELL, W., HAYTER, A. M., AND WOOD, w. 1983. Plant regeneration from callus of haploid immature barley embryos. Cer. Res. Comm., 11, 77-82.

THOMAS, M. R. AND SCOTT, K. J. 1985. Plant regeneration by somatic embryogenesis from callus initiated from immature embryos and immature inflorescences of Hordeum vulgare. J. Plant Physiol., 121, 159-169.

YATES, F. AND COCHRAN, W. G. 1938. The analysis of groups of experiments. J. Agric. Sci., 28, 556-580. 